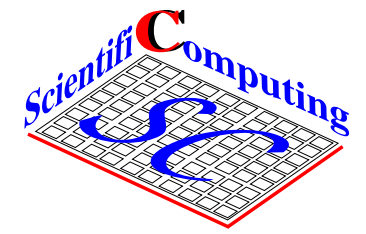

\title{
State-Space Representation of Instationary Two-Dimensional Airfoil Aerodynamics
}

\author{
Marcus Meyer, Hermann G. Matthies \\ Institute of Scientific Computing \\ Technical University Braunschweig \\ Brunswick, Germany
}

Informatikbericht Nr.: 2003-07

April 2003

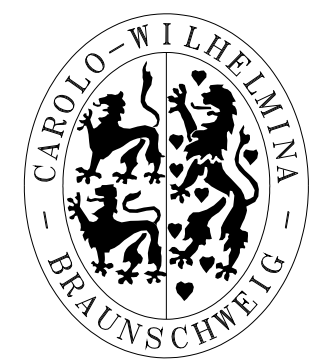




\title{
State-Space Representation of Instationary Two-Dimensional Airfoil Aerodynamics
}

\author{
Marcus Meyer, Hermann G. Matthies \\ Department of Mathematics and Computer Science \\ Technical University Braunschweig \\ Brunswick, Germany
}

Informatikbericht Nr.: 2003-07

April 2003

Contributed to Wind Engineering and Industrial Aerodynamics

\section{Location \\ Institute of Scientific Computing \\ Technical University Braunschweig \\ Hans-Sommer-Strasse 65 \\ D-38106 Braunschweig}

\section{Contact}

Phone: +49-(0)531-391-3000

Fax: +49-(0)531-391-3003

E-Mail: wiredtu-bs.de

www: http://www.tu-bs.de/institute/WiR

\section{Copyright}

(c)Institut für Wissenschaftliches Rechnen

Technische Universität Braunschweig

\section{Postal Address}

Institut für Wissenschaftliches Rechnen

Technische Universität Braunschweig

D-38092 Braunschweig

Germany 


\title{
State-Space Representation of Instationary Two-Dimensional Airfoil Aerodynamics
}

\author{
Marcus Meyer, Hermann G. Matthies \\ Institute of Scientific Computing \\ Technical University Braunschweig \\ Brunswick, Germany
}

\begin{abstract}
April 2003
Abstract

In the aero-elastic analysis of wind turbines based on blade element theory, the need to include a model of the local, two-dimensional instationary aerodynamic loads, commonly referred to as dynamic stall has become obvious in the last years. In this contribution we describe an alternative choice for such a model, based on [19]. Its derivation is governed by the flow physics, thus enabling interpolation between different profile geometries. To facilitate aeroelastic stability and sensitivity investigations, the original model is changed into state-space form, i.e. a system of differential equations. The transformation into state-space form is validated with numerical calculations.
\end{abstract}

Keywords: dynamic stall, state-space representation

\section{Contents}

1 Introduction 2

2 Modeling of Stationary Profile Coefficients 4

3 Modeling the Instationary Effects $\quad 7$

4 Transformation into State-Space Form $\quad 8$

5 Validation and Conclusions $\quad 11$ 


\section{Introduction}

For the numerical analysis of the dynamic behaviour of wind turbines in turbulent wind, the necessity and importance of including a model of the instationarity of the local two-dimensional flow, the so-called dynamic stall model, is widely recognised. As example the stationary versus the instationary profile coefficient for the lift as obtained in wind tunnel measurements are depicted in Fig.1, taken from [12]. The large differences are clearly visible. Thus only with a dynamic stall model can the aerodynamic forces in the linear as well as the stall regime be adequately modeled. Also aerodynamic damping properties and aeroelastic sta-

windtunnel measurements

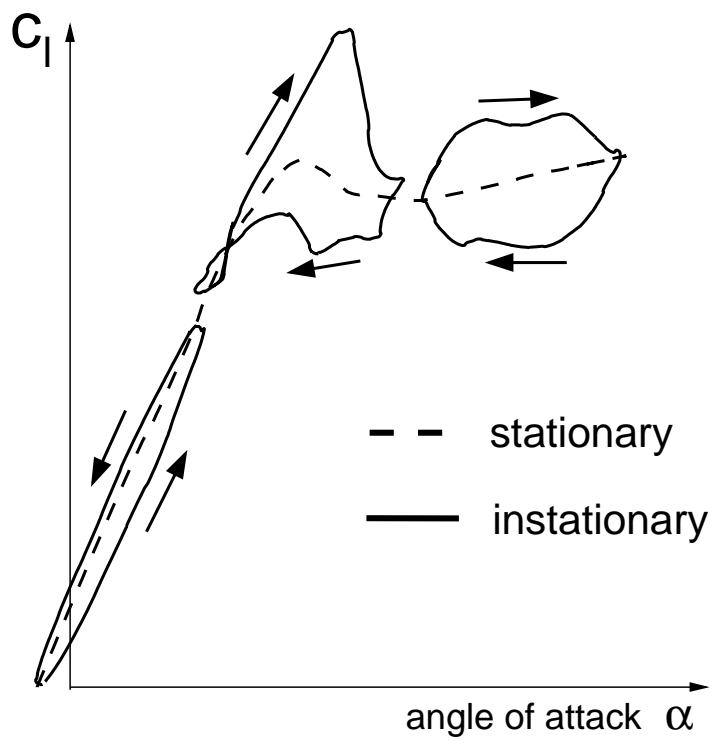

Figure 1: Stationary versus instationary lift coefficient from [12].

bility can only be investigated with the use of a proper model for the instationary aerodynamic loads. Although there exists an abundance of such dynamic stall models, ranging from simple to sophisticated, no model has yet succeeded over the other models in becoming something like a standard. In the literature we can find for example the model from LEISHMANN and BEDDOES [7], the ONERA [11] and DLR model [19], the models from GOMAN and KHRABROV [4] as well as TRUONG [18], which are mainly used for the analysis of helicopter dynamics, and the models from ØYE [21], RIS $\varnothing$ [14] and SNEL [15], mostly used in the wind turbine sector. For wind turbine applications we can define the following 
requirements for a successful dynamic stall model:

1. The derivation of the model should be based on the flow physics, since interpolations between different profile geometries are necessary and should yield meaningful results.

2. It has to be applicable for the whole range of angles of attack, $\alpha \in[-\pi, \pi]$.

3. In the limit of zero reduced frequency the stationary curves of the profile coefficients have to be reproduced.

4. In the linear regime it has to reproduce the results obtained by solving the Theodorsen equation [16].

5. The hysteresis curves in the critical stall regime have to be reproduced not only qualitatively, but also quantitatively, and the adaptation to measurements must be possible.

6. To be able to perform sensitivity and stability analyses, the model should be in state-space form, i.e. in the form of differential equations.

7. Last but not least it should be easy to implement and extend.

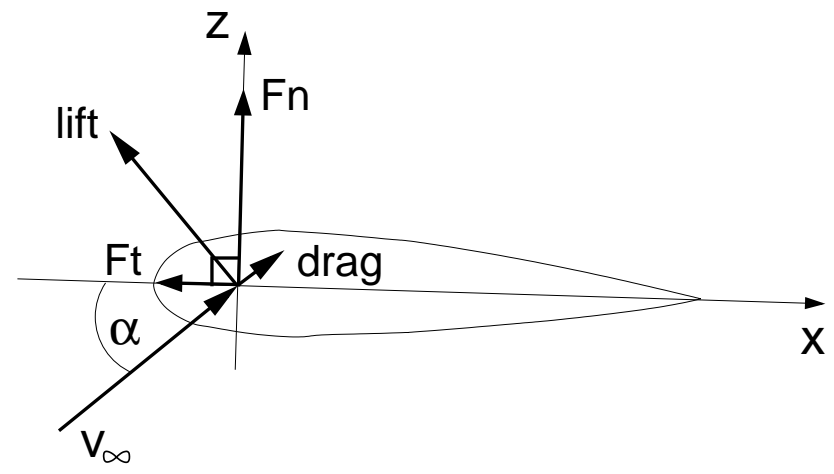

Figure 2: The profile coordinate system.

In a recent evaluation study by PETOT et al. [2], where different dynamic stall models were compared, the DLR [19] and ONERA [11] model gave the best results compared with measurements. In this study not only the prediction of instationary lift and drag coefficients, but also the prediction of the instationary moment coefficient was considered. This last requirement currently seems not very important for wind turbine applications, but as rotor blade size and flexibility increases, the prediction of the instationary moment coefficient will become more 
and more necessary. An advantage of the DLR model over the ONERA model is its inherent additive nature, i.e. it is easy to extend the model to include additional effects. At first sight a disadvantage of the DLR model might be the fact that it is not formulated as a set of differential equations. But we will show in the sequel that it is possible to transform the DLR model into state-space form, an essential prerequisite to perform sensitivity and stability analyses as well as model reduction of the aero-elastic system [10].

\section{Modeling of Stationary Profile Coefficients}

The model which we describe in the following is based on the work of BEDDOES [1], LEISHMAN and BEDDOES [6], as well as LEISS and WAGNER [9] and VAN DER WALL [19]. The starting point of the modeling process are the stationary profile coefficients for lift, drag and aerodynamic moment. In the past these have been measured in wind tunnel experiments, but nowadays also CFD calculations are giving increasingly reliable results. The first step is the transformation from the aerodynamic into the profile coordinate system by a rotation with the angle of attack $\alpha$, i.e. from lift and drag to normal force (perpendicular to the chord) and tangential force (along the chord), compare Fig.2. Thus we do not work with the standard lift and drag coefficients $c_{l}$ and $c_{d}$, but with a normal force coefficient $c_{n}$ and a tangential force coefficient $c_{t}$ :

$$
\begin{aligned}
c_{n} & =c_{l} \cos (\alpha)+c_{d} \sin (\alpha), \\
c_{t} & =c_{l} \sin (\alpha)-c_{d} \cos (\alpha) .
\end{aligned}
$$

The coefficient of the aerodynamic moment $c_{m}$ remains unchanged. The input of the stationary aerodynamic model are the non-dimensional flow velocity components in the profile coordinate system,

$$
\begin{aligned}
& \nu_{x}=\nu \cos (\alpha), \\
& \nu_{z}=\nu \sin (\alpha),
\end{aligned}
$$

with $\nu=v / v_{\text {ref }}$. Here $v$ is the absolute value of the free stream velocity and $v_{\text {ref }}$ a suitable reference velocity.

The mathematical model for the coefficients $c_{n}, c_{t}$ and $c_{m}$ consists of the superposition of different analytical functions, which describe the contributions of the attached and separated flow regions, depicted for the normal force coefficient in Fig.3. Each coefficient is described as the sum of the following functions,

$$
c_{k}\left(\nu_{x}, \nu_{z}, \boldsymbol{p}\right)=\sum_{i=1}^{3} c_{k, i}\left(\nu_{x}, \nu_{z}, \boldsymbol{p}\right), \quad k \in[t, n, m] .
$$




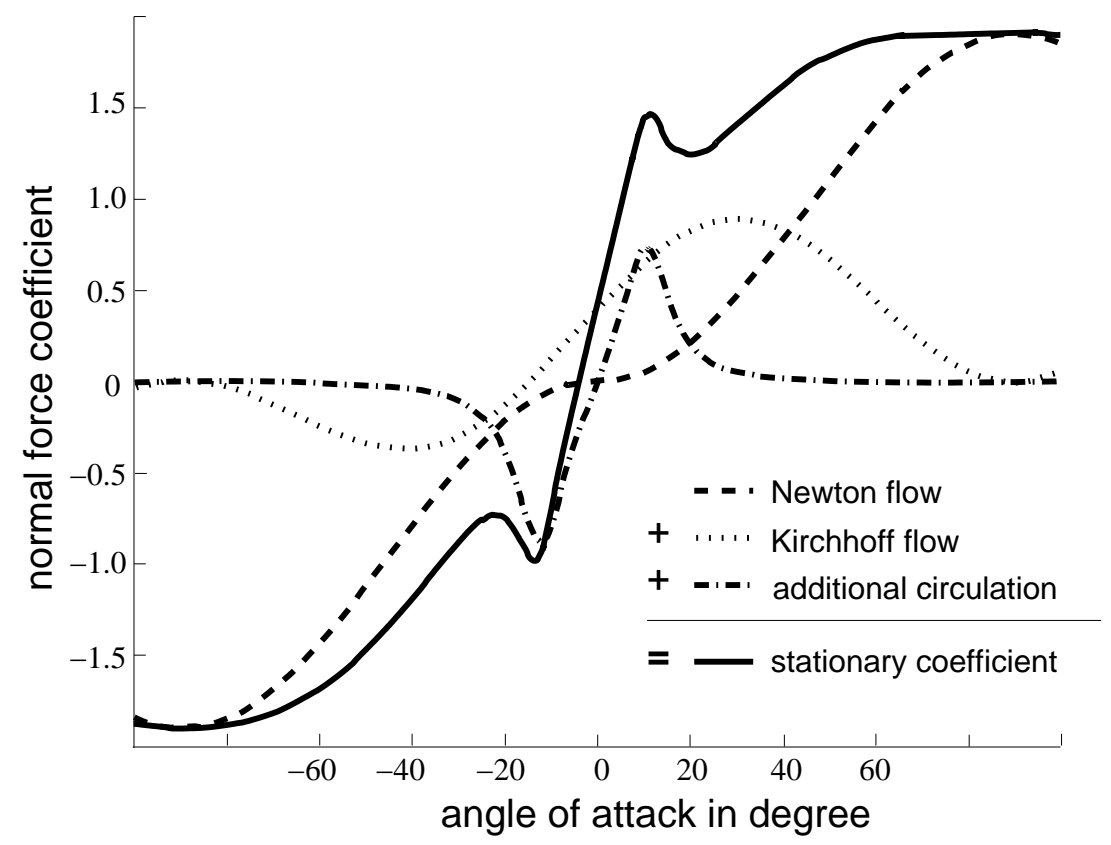

Figure 3: Stationary $c_{n}(\alpha)$ as superposition of analytical functions.

The vector $\boldsymbol{p}$ contains the parameters with which the analytical functions can be fitted to the measured coefficient curves using parameter optimisation algorithms [20].

The three functions $c_{k, i}$ are defined as follows [19]:

1. The first function describes the contribution of Newton's flow theory in the completely separated flow region at high angles of attack,

$$
\begin{aligned}
c_{t, 1} & =0, \\
c_{n, 1} & =p_{n 1} \frac{\nu_{z}\left|\nu_{z}\right|}{\nu^{2}}, \\
c_{m, 1} & =-p_{m 1} \frac{\nu_{z}\left|\nu_{z}\right|}{\nu^{2}} .
\end{aligned}
$$

2. The second function is based on the Kirchhoff potential flow theory [17] at moderately large angles of attack,

$$
\begin{aligned}
c_{t, 2} & =0 \\
c_{n, 2} & =p_{n 2} \frac{\left(\nu_{z}-p_{n 3}\right) \nu_{x}^{2}}{\nu^{3}} \\
c_{m, 2} & =-p_{m 2} \frac{\left(\nu_{z}-p_{m 3}\right) \nu_{x}^{2}}{\nu^{3}} .
\end{aligned}
$$


3. The third and most important function describes the contribution of the additional circulation at low angles of attack [19],

$$
c_{k, 3}=\left(c_{k}^{h k+}+c_{k}^{h k-}\right)+\left(c_{k}^{v k+}+c_{k}^{v k-}\right) .
$$

Here the first two terms define the contribution of trailing edge separation and the second two terms describe the contribution of leading edge separation, separately for positive and negative angles of attack. For the trailing edge separation we define the following function:

$$
c_{k}^{h k+}=\frac{\left|\nu_{x}\right|}{\nu^{2}} p_{k 4}^{+} p_{5}^{+} \Gamma_{k}^{h k+},
$$

with the normalised circulation function $\Gamma$ with a maximum at the stall angle of attack defined as

$$
\Gamma_{k}^{h k+}=\frac{p_{k 6}^{+}}{p_{k 6}^{+}+\left(\nu_{z}-p_{5}^{+}\right)^{2}} .
$$

The circulation at negative angles of attack is described in the same way, but with different values for the parameters $p_{j}$ in case of non-symmetric air-foils.

The second contribution to an increased circulation $c_{k}^{v k}$ might be caused by leading edge separation, depending on the air-foil shape. This is described by the following function:

$$
c_{k}^{v k+}=\frac{\left|\nu_{x}\right|}{\nu^{2}} p_{k 7}^{+} p_{5}^{+} \Gamma_{k}^{v k+}
$$

with the circulation function defined as

$$
\Gamma_{k}^{v k+}=\frac{\nu_{z}-p_{k 8}^{+}}{\left|p_{k 9}^{+}-p_{k 8}^{+}\right|} \frac{p_{k 10}^{+}}{p_{k 10}^{+}+\left(\nu_{z}-p_{k 9}^{+}\right)^{2}}
$$

The same functions are used for negative angles of attack, but again with different parameter values, if necessary. Depending on the air-foil shape it may be appropriate to model the positive stall without and the negative stall with this leading edge contribution.

Due to the additive form of the model the inclusion of more terms is easily accomplished: A forth function can for example model the influence of shedded vortices at high angles of attack, depending on Strouhal number with the inclusion of random effects, and a fifth function can be used to include 3D effects at the blade root sections. Another important advantage of the model is its firm grounding on the physics of aerodynamic flow theory. This means that the interpolation 
between different parameter sets for the different profile sections along the blade will give physically meaningful results. The optimal parameter values to fit the analytical curves to the measured profile coefficient curves can be obtained using numerical optimisation algorithms, in our case the application of the simplex method as described for example in [20] was sufficient.

\section{Modeling the Instationary Effects}

The extension of the stationary model described in the preceeding paragraph to include instationary effects is now relatively easy: According to the solution of a system of ordinary differential equations the input values $\nu_{x}$ and $\nu_{z}$ are modified to so-called effective values $\nu_{x}^{e f f}$ and $\nu_{z}^{e f f}$. Additionally the values of the parameters $p_{5}^{+/-}$in the circulation function (compare Eqs.(9) and (11)) are changed to the effective values $p_{5}^{+/-, e f f}=p_{5}^{+/-}+\Delta p$. In the original DLR model [19] these changes are calculated using a discrete approximation of the Duhamel integral. In the following we give a short description of the transformation into state-space form along the lines of LEISHMAN [8] and POIREL [13], which is better suited for our purposes, e.g. sensitivity and stability analyses and model reduction.

We start with an approximation of the Theodorsen function in the time domain, called the Wagner function $W(\tau)$ [5],

$$
W(\tau)=1-0.165 e^{-0.0455 \tau}-0.335 e^{-0.3 \tau}
$$

and the following approximation

$$
K(\tau)=1-0.5 e^{-0.13 \tau}-0.5 e^{-\tau}
$$

for the Küssner function [6]. Here $\tau$ is the non-dimensional time, defined as

$$
\tau=\frac{2 v}{c} t
$$

using the chord length $c$ of the profile and the absolute value of the free stream velocity $v$. The Wagner function describes the step response of the aerodynamic loads to changes of $\nu_{z}$, i.e. the change from $\nu_{z}$ to $\nu_{z}^{\text {eff }}$ and the Küssner function describes the change of $\nu_{x}$ to $\nu_{x}^{e f f}$. Thus these two functions describe the hysteresis curves of the instationary coefficients in the linear regime at low angles of attack. For the stall regime we use a linear ordinary differential equation (ODE) of first order [1], so the system response has the same frequency as the excitation,

$$
S(\tau)=1-A_{s} e^{-b_{s} \tau} .
$$


According to [19] the coefficient describing the time delay has the value $b_{s}=0.26$ and $A_{s}=1$. This function describes the change of the parameters $p_{5}^{+/-}$, i.e. the stall overshoot and the large hysteresis in the stall regime. Following [19] the input is defined as

$$
u_{s}=\dot{\nu}_{z}-\dot{\nu} \theta,
$$

where $\theta$ is the torsional displacement of the profile out of the initial position.

\section{Transformation into State-Space Form}

In the view of control theory all three functions $W(\tau), K(\tau)$ and $S(\tau)$ can be thought of as step response functions of the aerodynamic system for different inputs. The procedure to transform a step response function into state-space form can be found in most books on control theory, for example [3]: First we transform the generic step response in the time domain,

$$
W(t)=\left\{\begin{array}{cc}
1-A_{1} e^{-\frac{t}{T_{1}}}-A_{2} e^{-\frac{t}{T_{2}}} & \text { für } t \geq 0 \\
0 & \text { for } t<0
\end{array}\right.
$$

into the frequency domain using the Laplace transformation. We obtain

$$
W(s)=\frac{1}{s}-\frac{A_{1}}{s+\frac{1}{T_{1}}}-\frac{A_{2}}{s+\frac{1}{T_{2}}}
$$

with $T_{1}=\frac{c}{2 v b_{1}}$ and $T_{2}=\frac{c}{2 v b_{2}}$.

We need the transfer function $H(s)$ of the system, which is connected to the step response function $W(s)$ via multiplication with $1 / s$,

$$
W(s)=H(s) \frac{1}{s} .
$$

Thus our transfer function $H(s)$ is

$$
H(s)=s W(s)=1-\frac{A_{1} T_{1} s}{1+s T_{1}}-\frac{A_{2} T_{2} s}{1+s T_{2}} .
$$

For a transfer function in the form

$$
H(s)=\frac{b_{0}+b_{1} s+\ldots b_{n} s^{n}}{a_{0}+a_{1} s+\ldots a_{n} s^{n}},
$$

with $a_{n} \neq 0$ and at least one $b_{\nu} \neq 0$, the corresponding state-space form is

$$
\dot{\boldsymbol{x}}=\left(\begin{array}{ccccc}
0 & 1 & 0 & \ldots & 0 \\
0 & 0 & 1 & & 0 \\
& & & \ddots & \vdots \\
\vdots & \vdots & & & 1 \\
-\frac{a_{0}}{a_{n}} & -\frac{a_{1}}{a_{n}} & & \ldots & -\frac{a_{n-1}}{a_{n}}
\end{array}\right) \boldsymbol{x}+\left(\begin{array}{c}
0 \\
0 \\
\vdots \\
0 \\
\frac{1}{a_{n}}
\end{array}\right) u,
$$


with the output equation

$$
y=\left(b_{0}-a_{0} \frac{b_{n}}{a_{n}}, \quad \ldots, \quad b_{n-1}-a_{n-1} \frac{b_{n}}{a_{n}}\right) \boldsymbol{x}+\frac{b_{n}}{a_{n}} u .
$$

Now we can apply this transformation to our dynamic stall model. We obtain for the Wagner function

$$
\begin{aligned}
\dot{\boldsymbol{x}}_{w} & =\boldsymbol{A}_{w} \boldsymbol{x}_{w}+\boldsymbol{b}_{w} \nu_{z} \\
\nu_{z, \text { eff }} & =\boldsymbol{c}_{w}^{T} \boldsymbol{x}_{w}+d_{w} \nu_{z}
\end{aligned}
$$

with

$$
\begin{aligned}
& \boldsymbol{A}_{w}=\left(\begin{array}{cc}
0 & 1 \\
-b_{1 w} b_{2 w}(2 v / c)^{2} & -\left(b_{1 w}+b_{2 w}\right)(2 v / c)
\end{array}\right) \\
& \boldsymbol{b}_{w}=\left(\begin{array}{l}
0 \\
1
\end{array}\right) \\
& \boldsymbol{c}_{w}^{T}=\left(\frac{1}{2} b_{1 w} b_{2 w}(2 v / c)^{2} \quad\left(A_{1 w} b_{1 w}+A_{2 w} b_{2 w}\right)(2 v / c)\right) \\
& d_{w}=1-A_{1 w}-A_{2 w} \text {, }
\end{aligned}
$$

where $A_{1 w}=0.165, b_{1 w}=0.0455, A_{2 w}=0.335$ and $b_{2 w}=0.3$.

For the Küssner function we get similarly

$$
\begin{aligned}
\dot{\boldsymbol{x}}_{k} & =\boldsymbol{A}_{k} \boldsymbol{x}_{k}+\boldsymbol{b}_{k} \nu_{x} \\
\nu_{x, e f f} & =\boldsymbol{c}_{k}^{T} \boldsymbol{x}_{k}+d_{k} \nu_{x},
\end{aligned}
$$

with

$$
\begin{aligned}
& \boldsymbol{A}_{k}=\left(\begin{array}{cc}
0 & 1 \\
-b_{1 k} b_{2 k}(2 v / c)^{2} & -\left(b_{1 k}+b_{2 k}\right)(2 v / c)
\end{array}\right) \\
& \boldsymbol{b}_{k}=\left(\begin{array}{l}
0 \\
1
\end{array}\right) \\
& \boldsymbol{c}_{k}^{T}=\left(b_{1 k} b_{2 k}(2 v / c)^{2} \quad\left(A_{1 k} b_{1 k}+A_{2 k} b_{2 k}\right)(2 v / c)\right) \\
& d_{k}=0 \text {, }
\end{aligned}
$$

where $A_{1 k}=0.3, A_{2 k}=0.7, b_{1 k}=0.14$ and $b_{2 k}=0.53$.

For the stall function the state-space form is

$$
\begin{aligned}
\dot{x}_{s} & =A_{s} x_{s}+b_{s} u_{s} \\
\Delta p_{5} & =c_{s} x_{s}+d_{s} u_{s}
\end{aligned}
$$

with $A_{s}=b_{1 s}(2 v / c), b_{s}=1, c_{s}=p_{s} A_{1 s} b_{1 s} \frac{2 v}{c}, p_{s}=0.05$ and $d_{s}=0$. 
We can combine all three systems to form a block-diagonal system of ODEs in $\mathbb{R}^{5}$, which describes the instationary aerodynamic loads at the individual profile sections,

$$
\begin{aligned}
\dot{\boldsymbol{x}} & =\boldsymbol{S}_{A} \boldsymbol{x}+\boldsymbol{S}_{B} \boldsymbol{u}_{a}=\boldsymbol{g}\left(\boldsymbol{x}, \boldsymbol{u}_{a}, t\right) \\
\boldsymbol{y} & =\boldsymbol{S}_{C} \boldsymbol{x}+\boldsymbol{S}_{D} \boldsymbol{u}_{a}
\end{aligned}
$$

with the matrices

$$
\boldsymbol{S}_{A}=\left(\begin{array}{ccc}
\boldsymbol{A}_{w} & \mathbf{0} & \mathbf{0} \\
\mathbf{0} & \boldsymbol{A}_{k} & \mathbf{0} \\
\mathbf{0} & \mathbf{0} & A_{s}
\end{array}\right), \boldsymbol{S}_{B}=\left(\begin{array}{ccc}
\boldsymbol{b}_{w} & \mathbf{0} & \mathbf{0} \\
\mathbf{0} & \boldsymbol{b}_{k} & \mathbf{0} \\
\mathbf{0} & \mathbf{0} & b_{s}
\end{array}\right), \boldsymbol{u}_{a}=\left(\begin{array}{c}
\nu_{z} \\
\nu_{z} \\
\nu_{x} \\
\nu_{x} \\
u_{s}
\end{array}\right)
$$

and

$$
\boldsymbol{S}_{C}=\left(\begin{array}{ccc}
\boldsymbol{c}_{w}^{T} & \mathbf{0} & 0 \\
\mathbf{0} & \boldsymbol{c}_{k}^{T} & 0 \\
\mathbf{0} & \mathbf{0} & c_{s}
\end{array}\right), \quad \boldsymbol{S}_{D}=\left(\begin{array}{ccc}
\boldsymbol{d}_{w} & \mathbf{0} & 0 \\
\mathbf{0} & \boldsymbol{d}_{k} & 0 \\
\mathbf{0} & \mathbf{0} & d_{s}
\end{array}\right), \quad \boldsymbol{y}=\left(\begin{array}{c}
\nu_{z}^{\text {eff }} \\
\nu_{x}^{\text {eef }} \\
\Delta p_{5}
\end{array}\right)
$$

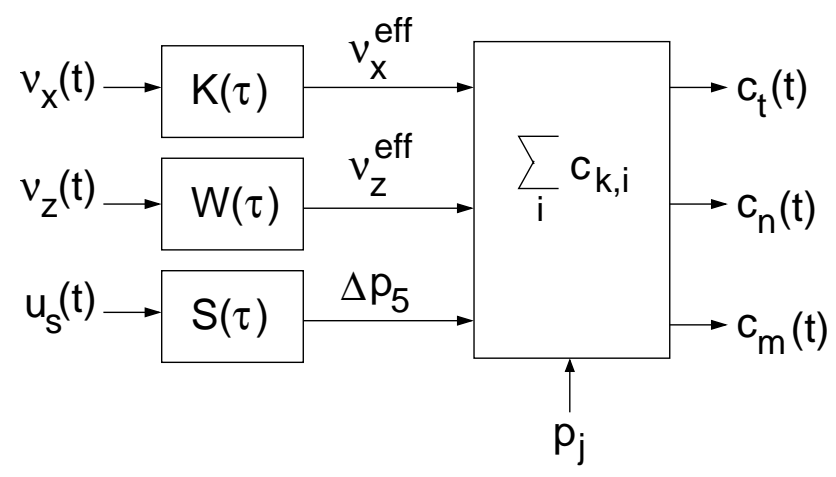

Figure 4: Block diagram of the dynamic stall model.

To summarise this dynamic stall model we can draw the block diagram as shown in Fig.4. The input values are the time histories of $\nu_{x}, \nu_{z}$ and $u_{s}$. Using the Küssner, Wagner and Stall functions we obtain the output values $\nu_{x}^{\text {eff }}$, $\nu_{z}^{\text {eff }}$ and $\Delta p_{5}$. These, together with the previously determined parameter values are the input values for the analytic functions, which in turn give as their output the instationary profile coefficients. 


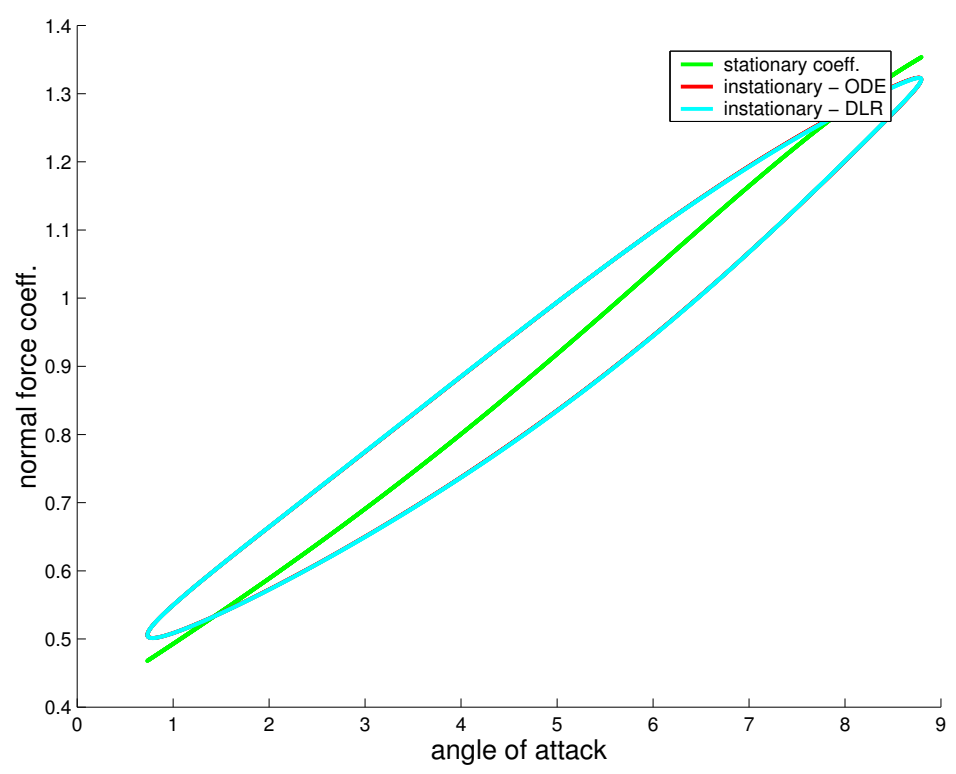

Figure 5: Linear regime.

\section{Validation and Conclusions}

To validate the transformation into state-space form, we compare the results of the modified DLR model in state-space form with results obtained using the original DLR [19] model at different angles of attack for the reduced frequency of $k=0.1$. The ODEs have been discretised with the second order accurate trapezoidal rule using a time step of 0.05 seconds. But also the discretisation with the first order accurate implicit and explicit Euler scheme gave satisfactory results. As example we show the results for the normal force coefficient in Figs.5, 6 and 7, and we can see a very good correspondence of the two models.

To conclude, we have shown an alternative choice for modeling the instationary two-dimensional aerodynamic loads. This model has been cast in the form of differential equations, suitable for the important tasks of aeroelastic stability and sensitivity analyses. We belive that due to its physical foundation and its additive nature it is very well suited for instationary wind turbine analysis. 


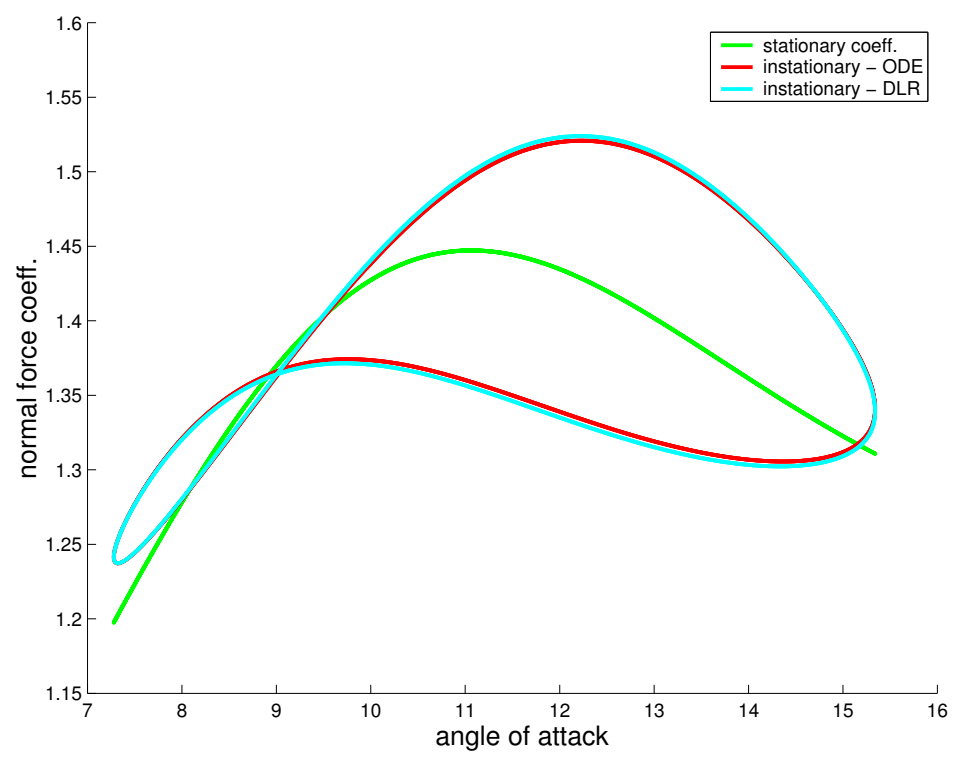

Figure 6: Stall regime

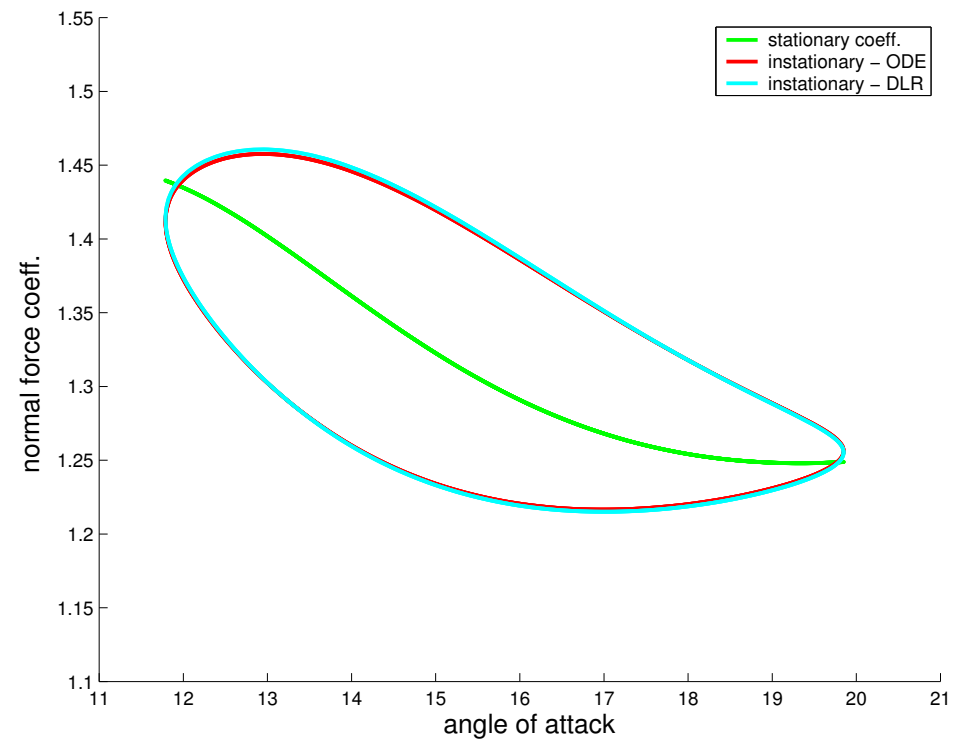

Figure 7: Post-Stall regime 


\section{References}

[1] T.S. Beddoes. A synthesis of unsteady aerodynamic effects including stall hysteresis. Vertica, 1:113-123, 1976.

[2] D. Petot et al. Stall effects and blade torsion - an evaluation of predictive tools. Journal of the American Helicopter Society, 10:320-331, 1999.

[3] O. Föllinger. Regelungstechnik. Hüthig Buch Verlag, Heidelberg, 1990.

[4] M. Goman and A. Khrabrov. State-space representation of aerodynamic characteristics of an aircraft at high angles of attack. Journal of Aircraft, 31(5):1109-1115, 1994.

[5] R.T. Jones. The unsteady lift of a wing of finite aspect ratio. Technical report, NACA Report 681, 1940.

[6] J.G. Leishman and T.S. Beddoes. A generalised model for airfoil unsteady aerodynamic behaviour and dynamic stall using the indicial method. In 42nd Annual Forum of the American Helicopter Society, pages 243-265, 1986.

[7] J.G. Leishman and T.S. Beddoes. A semi-empirical model for dynamic stall. Journal of the American Helicopter Society, 7:3-17, 1989.

[8] J.G. Leishman and K.Q. Nguyen. State-space representation of unsteady airfoil behaviour. AIAA Journal, 28(5):836-844, 1990.

[9] U. Leiss and S. Wagner. Toward a unified representation of rotor blade airloads with emphasis on unsteady and viscous effects. In Thirteenth European Rotorcraft Forum, 1987.

[10] M. Meyer. Reduktionsmethoden zur Berechnung des aeroelastischen Verhaltens von Windkraftanlagen. Braunschweiger Schriften zur Mechanik, Braunschweig, 2002.

[11] D. Petot. Differential equation modeling of dynamic stall. Rech. Aerosp., 5:59-72, 1989.

[12] R.A. Piziali. 2-D and 3-D oscillating wing aerodynamics for a range of angles of attack including stall. Technical report, NACA Technical Memorandum 4632, 1994.

[13] D.C. Poirel and S.J. Price. Post-instability behaviour of a structurally nonlinear airfoil in longitudinal turbulence. Journal of Aircraft, 34(5):619-626, 1997. 
[14] F. Rasmussen, J.T. Petersen, and H.A. Madsen. Dynamic stall and aerodynamic damping. Journal of Solar Energy Engineering, 121:150-155, 1999.

[15] H. Snel. Heuristic modeling of dynamic stall characteristics. In Proceedings of the EWEC, Dublin, pages 429-433, 1997.

[16] T. Theodorsen. General theory of aerodynamic instability and the mechanism of flutter. Technical report, NACA Report Nr. 496, 1934.

[17] B. Thwaites. Incompressible Aerodynamics. Oxford Clarendon Press, Oxford, 1960.

[18] V.K. Truong. Prediction of helicopter rotor airloads based on physical modeling of 3-D unsteady aerodynamics. Technical report, ONERA TP 1996-29, 1996.

[19] B. van der Wall. Analytische Formulierung der instationären Profilbeiwerte und deren Anwendung in der Rotorsimulation. DLR-FB 90-28, Deutsche Forschungsanstalt für Luft- und Raumfahrt, Braunschweig, 1990.

[20] W. Press et al. Numerical Recipies in C - The Art of Scientific Computing. Cambridge University Press, Cambridge, 1988.

[21] S. Øye. Dynamic stall simulated as time lag of separation. In Proceedings of the EWEC, 1994. 
1999-02 P. Niebert

1999-03 S. Eckstein, K. Neumann

1999-04 T. Gehrke, A. Rensink

2000-01 T. Kaiser, B. Fischer, W. Struckmann

2000-02 J. Saperia, J. Schönwälder

2000-03 A. Casties

2000-04 J. Koslowski

2000-05 S. Eckstein, P. Ahlbrecht, K. Neumann

2000-06 F. Strauß, J. Schönwälder, M. Mertens

2000-07 F. Strauß

2000-08 T. Gehrke, U. Goltz

2000-09 T. Firley

2001-01 K. Diethers

2002-01 R. van Glabbeek, U. Goltz

2002-02 J. Weimar

2002-03 H. G. Matthies, M. Meyer

2002-04 H. G. Matthies, J. Steindorf

2002-05 H. G. Matthies, J. Steindorf

2002-06 H. G. Matthies, J. Steindorf

2002-07 H. Firley, U. Goltz

2003-01 M. Meyer, H. G. Matthies

2003-02 C. Täubner

2003-03 T.-P. Fries, H. G. Matthies

2003-04 A. Keese, H. G. Matthies

2003-05 A. Keese, H. G. Matthies

2003-06 A. Keese

2003-07 M. Meyer, H. G. Matthies
A Temporal Logic for the Specification and Verification of Distributed Behaviour

Konzeptioneller Entwurf mit der Unified Modeling Language

A Mobile Calculus with Data

The Modula-2 Proving System MOPS

Policy-Based Enhancements to the SNMP Framework

Finite-Element-Interpolation der räumlichen Dichten eines Vielteilchensystems auf ungeordneten Gittern

A 2-dimensional view of the Chu-construction

Von parametrisierten Spezifikationen zu generierten Informationssystemen: ein Anwendungsbeispiel

JAX - A Java AgentX Sub-Agent Toolkit

Advantages and Disadvantages of the Script MIB Infrastructure

High-Level Sequence Charts with Data Manipulation

Regular languages as states for an abstract automaton

Tool-Based Analysis of Timed Sequence Diagrams

Well-behaved Flow Event Structures for Parallel

Composition and Action Refinement

Translations of Cellular Automata for Efficient

Simulation

Nonlinear Galerkin Methods for the Model Reduction of Nonlinear Dynamical Systems

Partitioned Strong Coupling Algorithms for

Fluid-Structure-Interaction

Partitioned but Strongly Coupled Iteration Schemes for Nonlinear Fluid-Structure Interaction

Strong Coupling Methods

Property Preserving Abstraction for Software

Verification

Efficient Model Reduction in Non-linear Dynamics

Using the Karhunen-Loève Expansion and

Dual-Weighted-Residual Methods

Modellierung des Ethylen-Pathways mit

UML-Statecharts

Classification and Overview of Meshfree Methods

Fragen der numerischen Integration bei stochastischen finiten Elementen für nichtlineare Probleme

Numerical Methods and Smolyak Quadrature for Nonlinear Stochastic Partial Differential Equations

A Review of Recent Developments in the Numerical Solution of Stochastic Partial Differential Equations (Stochastic Finite Elements)

State-Space Representation of Instationary

Two-Dimensional Airfoil Aerodynamics 